\title{
Neoparamoeba Page, 1987: light and electron microscopic observations on six strains of different origin
}

\author{
I. Dyková ${ }^{1, *}$, A. Figueras ${ }^{2}$, Z. Peric ${ }^{3}$ \\ ${ }^{1}$ Institute of Parasitology, Academy of Sciences of the Czech Republic, Branišovská 31, 37005 České Budějovice, Czech Republic \\ ${ }^{2}$ Concejo Superior de Investigaciones Cientificas, Instituto de Investigaciones Marinas, Eduardo Cabello 6, 36208 Vigo, Spain \\ ${ }^{3}$ Ministry of Agriculture and Fisheries, National Aquaculture Centre, Malta
}

\begin{abstract}
Although amoebic gill disease (AGD) has emerged as one of the most severe health problems in the fish industry, proof of the identity of AGD agents from various localities is still missing. Six strains of amoebae designated until recently as Paramoeba species (the agents of AGD) were studied in cultures by light and electron microscopy. Although they were isolated from gills of different hosts (Dicentrarchus labrax and Scophthalmus maximus) and from distant localities, their morphology was identical. The strains differed from Paramoeba eilhardi, the type species of the genus, in that they lacked the boat-shaped microscales on the cell surface but could be safely identified as belonging to the genus Neoparamoeba Page, 1987. Transmission electron microscopy revealed the presence of a symbiotic organism, Perkinsiella amoebae Hollande, 1980, in all strains under study. The only difference among the strains examined was found in the size of trophozoites, which could be attributed to the different origins of the strains, but until more refined diagnostic methods are available, in addition to $N$. pemaquidensis, the closely related species $N$. aestuarina also has to be taken into consideration as the agent of AGD.
\end{abstract}

KEY WORDS: Paramoeba $\cdot$ Neoparamoeba $\cdot$ Etiology of AGD $\cdot$ Mariculture Resale or republication not permitted without written consent of the publisher

\section{INTRODUCTION}

Over the past 15 yr more than 100 species of freeliving 'naked' amoebae have been determined from seawater and marine sediments. In the same period of time, free-living and commonly occurring amoebae of the long-known genus Paramoeba Schaudinn, 1896 emerged as causative agents of severe gill tissue damage in farmed salmonids Oncorhynchus mykiss, O. kisutch and Salmo salar (Kent et al. 1988, Roubal et al. 1989) and flatfishes Scophthalmus maximus (Dyková et al. 1995, 1998, 1999). Despite a concentrated effort, the identity of the agent which has been designated as Paramoeba sp., which causes amoebic gill

\footnotetext{
*E-mail: iva@paru.cas.cz
}

disease (AGD) of farmed fishes in distant parts of the world, remains uncertain. The most advanced research groups trying to control gill diseases of salmonids (Bryant et al. 1995, Findlay \& Munday 1998) have stressed the urgency of taxonomic studies in this direction.

As a first step, aimed at unravelling the biology and taxonomy of the agent of AGD, we have initiated comparative light and transmission electron microscopical studies of strains of different origin.

\section{MATERIALS AND METHODS}

In total, 7 'Paramoeba' strains of different origin from localities distant from each other were used in this study. The first 3 strains (P1, P2 and P10) were isolated 
from gills of 3 moribund larvae of sea bass Dicentrarchus labrax from a Mediterranean farm that experienced mortalities due to AGD. An additional 3 strains (SM53, SM57 and SM68) were isolated from gills of 3 turbots Scophthalmus maximus farmed in NW Spain (Dyková et al. 1999). One environmental strain (CCAP 1560/7) was obtained from the Culture Collection of Algae and Protozoa (Ambleside, UK) as a reference strain. Culture conditions, suitable methods, media and food supply recommended for marine gymnamoebae by Page (1983) and Tompkins et al. (1995) as well as the periodicity of subculturing were tested. Cultures were observed repeatedly in all phases of population growth. Light microscopic morphology as well as fine structure were studied during the long-term culturing to characterize and compare trophozoites of the strains included in the study. Morphological observations were made and morphometrics and locomotion rates determined on living trophozoites, in hanging drop preparations. The Feulgen reaction and Hoechst 33258 nucleic acid stain were applied to visualise the nuclei and so-called parasomes in trophozoites. The trophozoites of individual strains were measured several times during the long-term culturing. For this purpose the cultures which were in the best conditions were always selected. Twenty consecutive samples from sea bass strains were investigated in a transmission electron microscope and 17 samples were investigated from more recently obtained turbot strains. Clonal cultures of each of the strains were fixed for electron microscopy with $3 \%$ glutaraldehyde solution in $0.1 \mathrm{M}$ cacodylate or phosphate buffer and postfixed with $1 \%$ osmium tetroxide solution (Harris 1991). Each strain was maintained in parallel lines to ensure a continued supply of material for comparative studies.

Deposit of strains: Live or cryopreserved cultures are deposited in the Institute of Parasitology, Academy of Sciences of the Czech Republic, České Budějovice, Czech Republic.

\section{RESULTS}

\section{Isolation and laboratory cultures}

Since clonal culturing of individual strains was the basis for this comparative study, we can comment on our successful cloning procedure and maintenance of cultures in the laboratory: Of 2 basic techniques available, the agar plate method was the best one for growing Paramoeba strains. Non nutrient Bacto agar Difco (1.5 to $2 \%$ ) prepared with $75 \%$ seawater SIGMA was used. Primary isolates, i.e., the first groups of Paramoeba trophozoites which appeared in the vicinity of decaying gill tissue on the surface of agar plates, were transferred along with a piece of agar face down on non nutrient agar in new Petri dishes. They were moistened with $75 \%$ seawater (SIGMA). When starting to grow on the surface of agar plates, Paramoeba trophozoites could be recognized among other species of amoebae which originally colonized gills. Paramoeba trophozoites were the biggest, and especially those that formed dense growing zones around the inoculum had highly vacuolated cytoplasm (Fig. 1). The initial phase of subculturing was the most critical. Selection of the best group of trophozoites from each maternal culture and transfer to new agar plates had to be repeated a minimum of 10 times to purify the cultures; otherwise, there was a strong tendency for mixed populations of bacteria to overgrow them. In addition, ciliates and flagellates, which also originally colonized gills, survived for quite a long period of time in agar plate cultures. The gliding bacteria belonging to Cytophagaceas that were the cause of agar decomposition also accompanied primary isolates. When a certain degree of culture purification was achieved, natural food was substituted with autoclaved bacteria. Non nutrient agar was seeded with Pseudomonas sp. This species was selected as a food supply because it was the part of microflora associated with primary isolates of Paramoeba. Numerous consecutive transfers had to be made to obtain a homogeneous strain. Early attempts to establish cultures in liquid media while still contaminated with mixed populations of bacteria failed, as well as later attempts during the subculturing. Trophozoites survived in NMY75S liquid medium (Page 1983) for quite a long period of time, but they did not multiply greatly and were always much more endangered by bacterial overgrowth than agar plate cultures. Also, the handling of liquid cultures was more difficult due to the fact that trophozoite adhesion to the bottom of culture flasks was less firm than adhesion to the surface of agar, so even slight movements of liquid medium detached the trophozoites which transformed to floating forms. When transferred to liquid medium with small pieces of agar, trophozoites continued to be attached to the agar. Compared to other species of amoebae, clonal cultures were extremely difficult to obtain. Together with 1 Paramoeba trophozoite another small one belonging to a different species could easily be transferred to a new agar plate. We suppose that sometimes small trophozoites of other species may be engulfed. This, along with difficulties initiating the multiplication of single trophozoites, was the reason why many attempts to isolate the clones failed. Agar plate cultures were maintained in an incubator at $20^{\circ} \mathrm{C}$ and subcultured regularly at intervals of 4 to $5 \mathrm{~d}$ depending on the degree of culture purification. When moistened adequately with $75 \%$ seawater, monoxenic cultures could be subcultured at $1 \mathrm{wk}$ intervals. Longer 


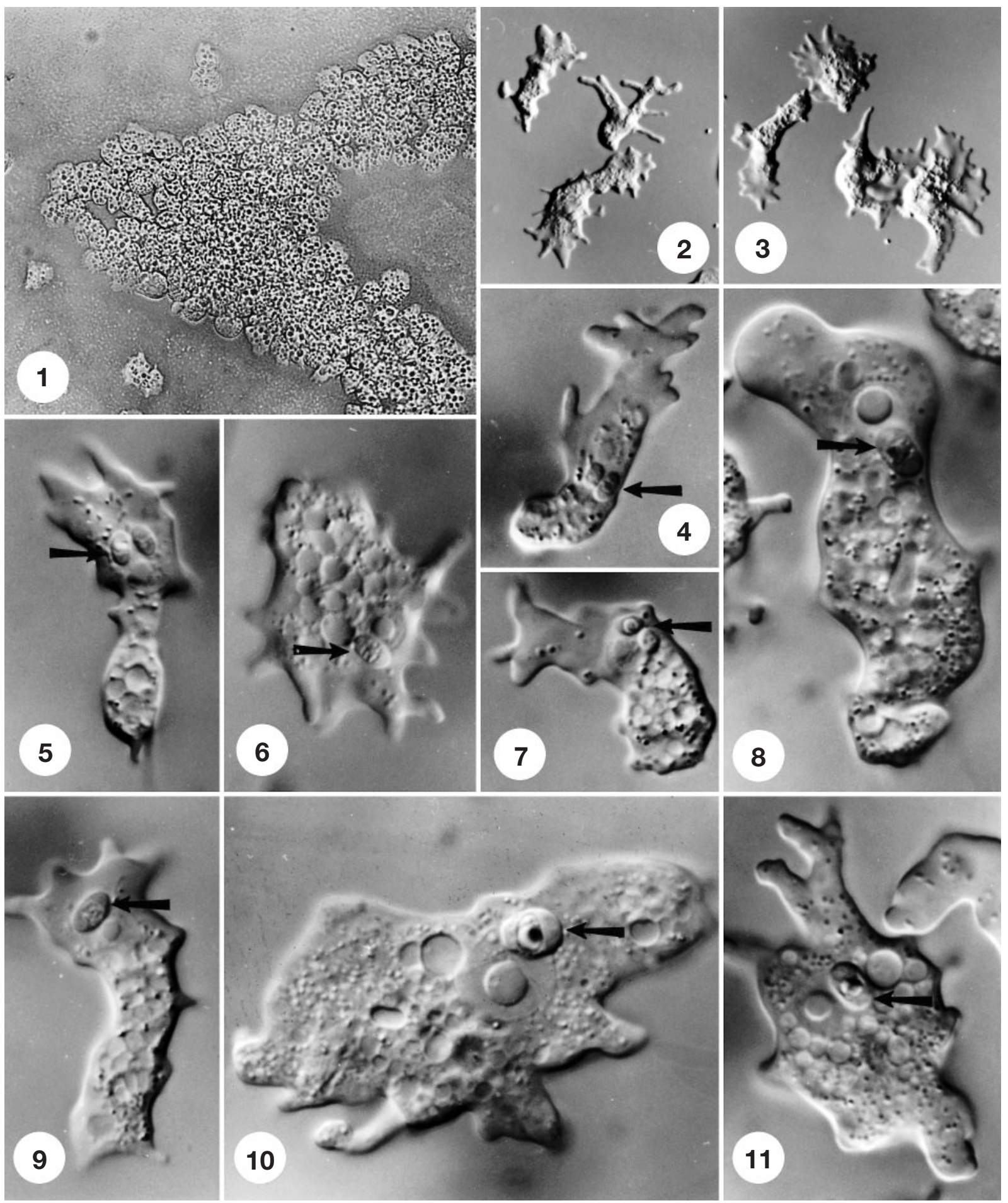

Figs. 1 to 11. Trophozoites of Neoparamoeba strains of different origin. The Olympus Nomarski DIC system. Arrows indicate the presence of symbiont Perkinsiella amoebae in the cytoplasm of trophozoites. Fig. 1. Neoparamoeba agar plate culture (strain P1), dense growing zone as seen through Petri dish. Figs. 2 \& 3. Neoparamoeba strain isolated from gills of Scophthalmus maximus (SM68), trophozoites in hanging drop preparation, $\times 540$. Figs. 4, 5, 7 \& 9. Trophozoites of Neoparamoeba strain (SM57) isolated from gills of $S$. maximus, $\times 1700$. Fig. 6. Trophozoite of environmental strain CCAP 1560/7, ×1700. Figs. 8, 10 \& 11. Mediterranean Neoparamoeba strain (P1) isolated from gills of Dicentrarchus labrax,$\times 1700$ 
periods of time negatively affected the viability of trophozoites. Monoxenic clonal cultures that became dense enough were subcultured by washing them with seawater and transferring them to new agar plates (1 to 2 plates). Several culture failures were experienced, almost always due to overgrowth with bacteria. Nevertheless, with the exception of 1 strain, we managed to maintain clonal cultures continuously for almost $2 \mathrm{yr}$. In ageing clonal cultures, shrunk elongated trophozoites appeared. Such cultures could be recovered by treating them as at the beginning of subculturing, i.e., selecting the best inocula for each of the following passages.

The following conditions were found to be optimal for cryopreservation of clonal cultures: Trophozoites were washed off the surface of agar plate with $2 \mathrm{ml}$ of $75 \%$ seawater (SIGMA), then the mixture of $3 \mathrm{ml}$ of $75 \%$ seawater and $0.3 \mathrm{ml}$ of cryoprotectant DMSO (SIGMA) was added and the volume was adjusted with sea water to $6 \mathrm{ml}$. Six cryotubes containing $1 \mathrm{ml}$ of trophozoite suspension each were equilibrated $30 \mathrm{~min}$ at room temperature. Afterwards they were exposed for $24 \mathrm{~h}$ to the liquid nitrogen vapours and finally placed in liquid nitrogen. The viability of trophozoites was always tested immediately after cryopreservation using the content of 1 cryotube; the rest were stored in cryocollection.

\section{Light microscopy}

The light microscopic morphology of trophozoites of all strains under study was characteristic of the genus Paramoeba as described by Page (1970, 1973, 1983), Kent et al. (1988) and Dyková et al. (1999). There were no differences among individual strains in the morphology of the trophozoites maintained in liquid medium or on agar plates (Figs. 2 to 11). Differences in the size of trophozoites divided our strains into 2 groups. Two Mediterranean strains (Figs. 8, 10 \& 11) had trophozoites with the range of average length exceeding $30 \mu \mathrm{m}$ (31.2 to $37.5 \mu \mathrm{m})$, while a third Mediterranean strain and 3 strains from turbots (Figs. 4, 5, 7 \& 9) had smaller trophozoites with the range of average length not attaining $30 \mu \mathrm{m}$ (18.3 to $26.1 \mu \mathrm{m})$. Slight differences in measurements recorded during the long-term culturing did not change the rate of size between the 2 groups of strains. Cysts were never observed in cultures of our strains, and the type of floating stages was always the same. In trophozoites of all strains the socalled parasome, a symbiotic organism for which Hollande (1980) established the taxon Perkinsiella amoebae, was found. This organism was easily discernible in live trophozoites when observed in Nomarski DIC (see arrows in Figs. 4 to 11). In the initial phase of cul- turing, the Feulgen reaction visualised the presence of more than 1 'parasome' in numerous trophozoites. A maximum of 6 'parasomes' in 1 cell was detected.

\section{Transmission electron microscopy}

No discernible differences were found in the ultrastructural organisation of the strains under study. The cell surface of trophozoites from agar plate cultures was coated mostly with thin amorphous glycocalyx (Figs. 12 to 17). The differentiation of glycocalyx was observed rather exceptionally. The hair-like glycostyles were sometimes observed on the invaginated cell surface in food vacuoles. The cytoplasm, with food vacuoles and generally distributed pinocytotic vesicles, contained spherical or ovoid mitochondria with slightly branched tubular cristae. The Golgi complex was located in the paranuclear zone (Fig. 17). The endoplasmic reticulum was not extensive in the cytoplasm. A large nucleus with finely granular matrix contained single nucleolus (Fig. 12). Numerous nuclear pores with electron-dense material traversed the nuclear membrane (Fig. 17). The shape of the nucleus was polymorphic, and was modified by the presence of the symbiotic organism Perkinsiella amoebae Hollande, 1980. The symbionts were always closely associated with the amoeba nucleus, which sometimes formed lobes or enveloped part of this organism (Figs. 14 \& 16). In all samples investigated, the same symbiotic organisms undergoing the same stages of development were observed. The same sequence of developmental stages was found in trophozoites of all strains; however, division was observed only exceptionally. As far as can be judged from the hitherto published micrographs, P. amoebae observed by us was almost identical with that reported in previous papers (Grell \& Benwitz 1970, Perkins \& Castagna 1971, Hollande 1980).

\section{DISCUSSION}

Since the first conclusive report on the aetiology of AGD in Coho salmon in Kent et al. (1988), who presented the agent as Paramoeba pemaquidensis Page, 1970, the same specific name has been adopted in the following papers. One year before, Page (1987) published a classification of naked amoebae of the phylum Rhizopoda in which he restricted the genus Paramoeba to species which have trophozoites with a surface covered with microscales as found in the type species P. eilhardi Schaudinn, 1896. P. pemaquidensis Page, 1970 as well as $P$. aestuarina Page, 1970 were transferred by Page (1987) to the newly established genus Neoparamoeba as N. pemaquidensis (Page, 1970) (the 

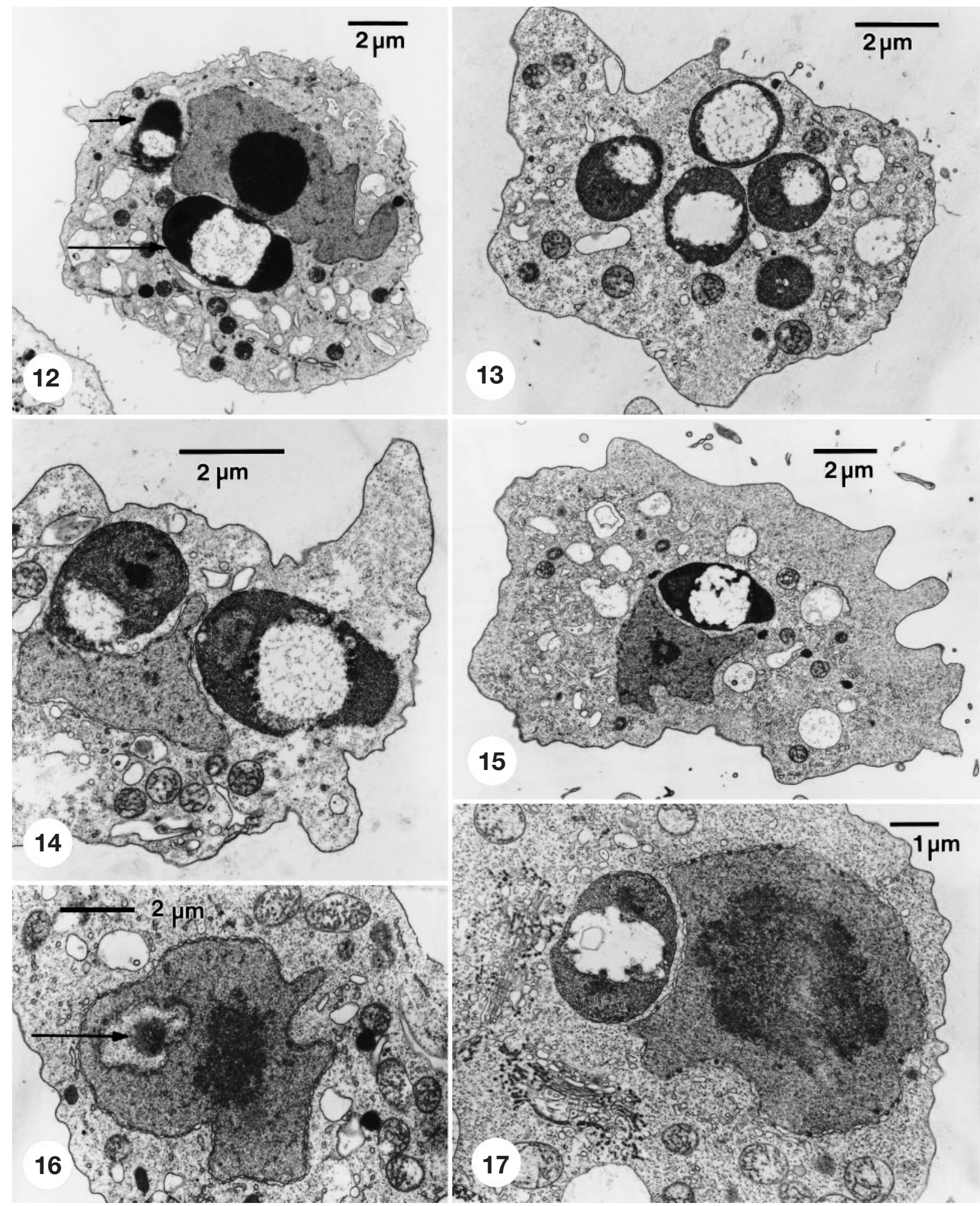

Figs. 12 to 17. Transmission electron micrographs of Neoparamoeba trophozoites to show characteristics of the genus. Figs. 12 to 14. Ultrathin section through the trophozoite of Mediterranean strain (P1) isolated from gills of Dicentrarchus labrax. Fig. 12. Vacuolated cytoplasm contains relatively small mitochondria, large lobose nucleus and 2 symbionts in different phase of their development, which are situated in the close vicinity of the nucleus. Fig. 13. Five symbionts in the cytoplasm of trophozoite sectioned out of the level of nucleus. Fig. 14. Two symbionts situated in the close vicinity of the nucleus modify its shape. Figs. 15 \& 16. Ultrastructure of 2 strains isolated from gills of Scophthalmus maximus (SM57 and SM68). Fig. 15. Overview of trophozoite ultrastructure with predominating symbiont situated in the close vicinity of the nucleus. Fig. 16. The union of nucleus and symbiont (arrow) that is encompassed by nucleoplasm. Fig. 17. Trophozoite of strain SM68 isolated from gills of $S$. maximus showing 
type species), and N. aestuarina (Page, 1970). The cell surface of Neoparamoeba species was characterised by dense glycocalyx about $10 \mathrm{~nm}$ thick. Hair-like filaments observed on the surface coat of AGD agents (Kent et al. 1988, Dyková et al. 1995) were not mentioned in the generic diagnosis of Neoparamoeba. The importance of cell surface structures for the taxonomy of amoebae was stressed in several genera. The presence of boat-shaped microscales on the surface of $P$. eilhardi as documented by Grell \& Benwitz (1966, 1970) undoubtedly separates this species from species described thus far as agents of AGD. In addition, we found the basic morphological features of the strain CCAP $1560 / 7$ as listed in the Catalogue of Strains (Tompkins et al. 1995) as N. aestuarina Page, 1987 (formerly as $P$. aestuarina) to be identical with other strains under study. Until a more refined taxonomic revision is available, the reasons mentioned above should be considered important enough to respect the taxonomic proposal of Page (1987). Consequently, the agents of AGD should be assigned to Neoparamoeba. The allotment of Paramoeba sp. and Dactylamoeba spp. (with scales on the surface) along with Mayorella spp. (with the surface cuticle) to the same family, i.e. Paramoebidae, seems to be slightly artificial. The same applies to the grouping of Neoparamoeba spp. with Vexillifera spp. in the family Vexilliferidae. It is necessary to compare the surface scales in the species $P$. eilhardi, Dactylamoeba spp. (D. stella and D. bulla) and Korotnevella nivo Smirnov, 1996/1997. This is one way to verify the legitimacy of Smirnov's suggestion (1996/1997), who proposed replacing the generic name Dactylamoeba with the newly coined name Korotnevella. The scales in these amoebae are pivotal features much like in the genus Cochliopodium. A detailed discussion of this problem, however, is beyond the scope of this paper. Shifting genera between families is less important for a proper etiological diagnosis than the use of a correct species name. In this case it is appropriate to adhere to Page's (1987) proposal.

Since the description of the Paramoeba type species in the past century, the so-called parasome has been assumed to be the main diagnostic feature of the genus. This intracellular structure, originally thought to be a cell organelle or secondary nucleus, has also been described in other species: $P$. perniciosa, the agent of 'gray crab disease' (Perkins \& Castagna 1971), and $P$. chaetognathi Grassi, 1881 and P. pigmentifera Grassi, 1881, parasites of Spadella spp. (Chaetognatha) which were later assigned to the independent genus Janickina Chatton, 1953 which belongs to the same family, Paramoebidae (Chatton 1953). Being described in 3 different genera of amoebae belonging to 2 different families, the so-called parasome, i.e., Perkinsiella amoebae Hollande, 1980, which lives as a symbiont in the cytoplasm, can hardly be considered the main diagnostic feature.

Although nothing is known about the metabolic association between Paramoeba spp. and Perkinsiella amoebae, their union along with the fact that in ageing cultures the number of trophozoites with fully developed Perkinsiella amoebae decreased support the idea of an inseparable partnership of vital importance.

The experience acquired during our long-term culturing of Paramoeba strains corresponds to some conclusions made by Martin (1985). The attachment of trophozoites to substrate is a basic condition for population growth. Testing the population growth in stationary and suspension cultures, Martin recognised that amoebae grew well only in stationary cultures.

Although we did not apply special methods to visualise glycocalyx in our TEM studies, we share the opinion of Kent et al. (1988), who recognised slight differences in the morphology of cell coat linked with culture characteristics. This was confirmed by Chávez et al. (1986), who found that culture conditions had an effect on the development of the cell coat: an amorphous cell coat 67 to $182 \mathrm{~nm}$ thick that was lacking in axenically grown amoebae covered the surface of those that were grown with bacteria.

Acknowledgements. This research was funded by the Grant Agency of the Academy of Sciences of the Czech Republic (Project No. A6022802) and by the Grant Agency of the Czech Republic (Project No. 206/00/0265).

\section{LITERATURE CITED}

Bryant MS, Lester RJG, Whittington RJ (1995) Immunogenicity of amoebic antigens in rainbow trout, Oncorhynchus mykiss (Walbaum). J Fish Dis 18:9-19

Chatton E (1953) Classe des Lobosa Leidy, 1879. Ordre des Amoebiens nus au Amoebaea. In: Grassé PP (ed) Traité de Zoologie Tom I, Fasc. II. Masson et Cie, Paris, p 42-46

Chávez LA, Balamuth W, Gong T (1986) A light and electron microscopical study of a new, polymorphic free-living amoeba, Phretamoeba balamuthi n. g., n. sp. J Protozool 33:397-404

Dyková I, Figueras A, Novoa B (1995) Amoebic gill infection of turbot, Scophthalmus maximus. Folia Parasitol 42:91-96

Dyková I, Figueras A, Novoa B, Fernandez-Casal J (1998) Paramoeba sp., an agent of amoebic gill disease of turbot Scophthalmus maximus. Dis Aquat Org 33:137-141

Dyková I, Figueras A, Novoa B (1999) Epizooic amoebae from the gills of turbot Scophthalmus maximus. Dis Aquat Org 38:33-39

Findlay VL, Munday BL (1998) Further studies on acquired resistance to amoebic gill disease (AGD) in Atlantic salmon, Salmo salar L. J Fish Dis 21:121-125

Grell KG, Benwitz G (1966) Die Zellhülle von Paramoeba eilhardi Schaudinn. Z Naturforsch 21B:600-602

Grell KG, Benwitz G (1970) Ultrastruktur mariner Amöben I. Paramoeba eilhardi Schaudinn. Arch Protistenkd 112: 119-137

Harris R (ed) (1991) Electron microscopy in biology. OIRL 
Press at Oxford University Press, Oxford

Hollande A (1980) Identification du parasome (Nebenkern) de Janickina pigmentifera a un symbionte (Perkinsiella amoebae nov. gen. - nov. sp.) apparenté aux flagellés kinetoplastidiés. Protistologica 16:613-625

Kent ML, Sawyer TK, Hedrick RP (1988) Paramoeba pemaquidensis (Sarcomastigophora: Paramoebidae) infestation of the gills of coho salmon Oncorhynchus kisutch reared in seawater. Dis Aquat Org 5:163-169

Martin RE (1985) Population growth in stationary and suspension culture of Paramoeba pemaquidensis Page (Amoebida, Paramoebidae). J Protozool 32:738-739

Page FC (1970) Two new species of Paramoeba from Maine. J Protozool 17:421-427

Page FC (1973) Paramoeba: a common marine genus. Hydrobiologia 41:183-188

Page FC (1983) Marine Gymnamoebae. Institute of Terrestrial Ecology, Culture Centre of Algae and Protozoa, Cambridge

Editorial responsibility: Wolfgang Körting,

Hannover, Germany
Page FC (1987) The classification of 'naked' amoebae of phylum Rhizopoda. Arch Protistenkd 133:199-217

Perkins FO, Castagna M (1971) Ultrastructure of the Nebenkörper or 'secondary nucleus' of the parasitic amoeba Paramoeba perniciosa (Amoebida, Paramoebidae). J Invertebr Pathol 17:186-193

Roubal FR, Lester RJG, Foster CK (1989) Studies on cultured and gill attached Paramoeba sp. (Gymnamoebae: Paramoebidae) and the cytopathology of paramoebic gill disease in Atlantic salmon, Salmo salar L., from Tasmania. J Fish Dis 12:481-493

Smirnov AV (1996/1997) Two new species of marine amoebae: Hartmannella lobifera n. sp. and Korotnevella nivo n. sp. (Lobosea, Gymnamoebida). Arch Protistenkd 147: 283-292

Tompkins J, De Ville MM, Day JG, Turner MF (eds) (1995) Media recipes. In: Catalogue of strains 1995. Natural Environment Research Council, Ambleside

Submitted: June 19, 2000; Accepted: September 12, 2000 Proofs received from author(s): October 30, 2000 